\title{
Dirac equation based on the vector representation of the Lorentz group
}

\author{
Eckart Marsch $^{1, \mathrm{a}}{ }_{\mathbb{D}}$, Yasuhito Narita ${ }^{2, \mathrm{~b}}$ \\ ${ }^{1}$ Extraterrestrial Physics Group, Institute for Experimental and Applied Physics, Christian Albrechts \\ University at Kiel, Leibnizstraße 11, 24118 Kiel, Germany \\ 2 Space Research Institute, Austrian Academy of Sciences, Schmiedlstraße 6, 8042 Graz, Austria
}

Received: 22 July 2020 / Accepted: 22 September 2020 / Published online: 6 October 2020

(C) The Author(s) 2020

\begin{abstract}
In this paper, we derive an expanded Dirac equation for a massive fermion doublet, which has in addition to the particle/antiparticle and spin-up/spin-down degrees of freedom explicity an isospin-type degree of freedom. We begin with revisiting the four-vector Lorentz group generators, define the corresponding gamma matrices and then write a Dirac equation for the fermion doublet with eight spinor components. The appropriate Lagrangian density is established, and the related chiral and $S U(2)$ symmetry is discussed in detail, as well as applications to an electroweak-style gauge theory. In "Appendix," we present some of the relevant matrices.
\end{abstract}

\section{Introduction}

The goal of this study is to reconsider an old subject, namely the Lorentz group and its representations [1-4], yet here with emphasis on the four-vector generators of the Lorentz group. The new result will be an extended Dirac equation [5] for a massive fermion doublet which has another intrinsic degree of freedom related to $S U(2)$ symmetry. The long history of the Dirac and related equations has been reviewed some years ago by Esposito [6]. In the present context, we just mention that ideas similar than ours were discussed long time ago in the paper titled "Fermions without spinors" [7], whereby the authors used the classical Kähler tensor field equation [8], which was extended by them and then applied to fermions.

In that paper, extensive use was made of differential forms and calculus and of the related advanced mathematics which even today is not a standard tool of theoretical physics or modern quantum field theory, as it is presented in text books [9-11] on the standard model (SM) of elementary particle physics. The attempts to establish extended Dirac equations continue until day, including recently also color [12] as an intrinsic degree of freedom of a fermion. The work by Kerner [13] provides a concise introduction to this subject. However, the algebraic effort in that theory is substantial, and the equation derived is mathematically more sophisticated than the original Dirac equation.

In our paper, we remain modest and stay closer to the mathematical language used in the SM as described in the excellent textbook by Schwartz [11] published only a few years

\footnotetext{
a e-mail: marsch@physik.uni-kiel.de (corresponding author)

b e-mail: yasuhito.narita@oeaw.ac.at
} 
ago. When establishing here an extended Dirac wave equation, we aim thereby at least algebraic complexity and most physical transparency. Of course, any prediction made by this equation ultimately has to face experimental reality that decides about its validity. Particles transforming according to various Lorentz group representations are endowed with different physical properties and thus will respond differently to gauge fields, e.g., like that of electromagnetism. In the massless case, the new equation can be naturally disassembled into four Weyl fields, two for each of the up- and down-components of the bi-spinor corresponding to the fermion doublet.

The outline of our paper is as follows: We start with revisiting the Lorentz transformation in Minkowski space and consider the related generators of the Lorentz group. These generators obey the Lorentz algebra, which in its simplest representation yields the Dirac equation. We will instead consider the four-vector generators of the Lorentz group. They act instead of Pauli spinors on complex four-component vectors which describe two more degrees of freedom corresponding to an isospin doublet. It may correspond to flavor as used in the SM as referring or corresponding to the up- and down-Dirac spinors of the Standard Model (SM) doublets of the three generations, respectively, of leptons and quarks. We then derive the corresponding extended Dirac equation for the fermion doublet and discuss its properties, and in particular chiral and $S U$ (2) symmetry. We also describe applications to an electroweakstyle gauge theory. Some relevant matrices are presented in "Appendix." Finally, we present our conclusions.

\section{The four-vector Lorentz group generators}

The purpose of this partially tutorial section is to elucidate the connections between the generators of the Lorentz group (LG) in spinor and vector representation. It is well known that the Lie algebra for the Lorentz group [1-4] can be decomposed into two commuting and thus independent subalgebras, such that $s o(3,1)=s u(2) \oplus s u(2)$. They define the generators of the irreducible $S U(2) \oplus S U$ (2) representation of the LG. The four-vector LG generators can be assembled in a tensor in Minkowski space-time and be written explicitly in $4 \times 4$ matrix form as

$$
L^{\mu \nu}=\left(\begin{array}{cccc}
0 & K_{x} & K_{y} & K_{z} \\
-K_{x} & 0 & J_{z} & -J_{y} \\
-K_{y} & -J_{z} & 0 & J_{x} \\
-K_{z} & J_{y} & -J_{x} & 0
\end{array}\right) .
$$

Here, we introduced the standard Hermitian rotation operator $\mathbf{J}=\left(J_{x}, J_{y}, J_{z}\right)$ and the anti-Hermitian boost operator $\mathbf{K}=\left(K_{x}, K_{y}, K_{z}\right)$. These four-vector generators of the LG are $4 \times 4$-matrix three-vectors in real space. The matrices are quoted for completeness in "Appendix." According to their definitions, the rotation and boost operators obey the wellknown linked three-vector equations of the Lorentz algebra, which can be written concisely as $\mathbf{J} \times \mathbf{J}=i \mathbf{J}, \quad \mathbf{K} \times \mathbf{K}=-i \mathbf{J}, \quad \mathbf{J} \times \mathbf{K}=\mathbf{K} \times \mathbf{J}=i \mathbf{K}$, where the cross-product sign stands for the commutator $[$,$] . We can then define the following linear combinations$

$$
\mathbf{J}_{ \pm}=\frac{1}{2}(\mathbf{J} \pm i \mathbf{K}),
$$

which obey the corresponding relations

$$
\mathbf{J}_{ \pm} \times \mathbf{J}_{ \pm}=i \mathbf{J}_{ \pm}, \mathbf{J}_{ \pm} \times \mathbf{J}_{\mp}=0 .
$$


These commutation relations are constitutive for the Lie algebra $s o(3,1)=\operatorname{su}(2) \oplus \operatorname{su}(2)$ of the LG and signify that it can be decomposed into two commuting $s u(2)$ subalgebras determining the generators of the $S U(2)$ group.

At this point, we recall that in the Dirac equation [5,9-11] the two simplest possible spinor representations of the LG are employed. They are given by the generator pairs $\mathbf{J}=\frac{1}{2} \boldsymbol{\sigma}$ and $\mathbf{K}= \pm \frac{i}{2} \sigma$, which obey the above vector algebra and are based on the fundamental representation of $S U(2)$ as given by the Pauli matrix vector $\sigma$ [14]. Then, either $\mathbf{J}_{+}=\mathbf{J}$, and the trivial one is $\mathbf{J}_{-}=0$ or vice versa, which are just the two well-known $\left(\frac{1}{2}, 0\right)$ or $\left(0, \frac{1}{2}\right)$ asymmetric representations of the LG.

In contrast, we will make use of the symmetric and constitutive original four-vector representation of the LG, the independent generators of which are defined by the matrix operator $\mathbf{J}_{ \pm}=\frac{1}{2} \boldsymbol{\Sigma}_{ \pm}$, with $\mathbf{J}_{ \pm}^{2}=s(s+1) 1_{4}$ and $s=\frac{1}{2}$. Here, $1_{4}$ means the $4 \times 4$ unit matrix (and similarly $1_{2}$ the $2 \times 2$ unit matrix). We may call $\mathbf{J}_{+}$the right-chiral, respectively, $\mathbf{J}_{-}$the left-chiral spin operator, involving the generalized $4 \times 4$ spin matrices,

$$
\begin{gathered}
\Sigma_{ \pm x}=\left(\begin{array}{cccc}
0 & \pm 1 & 0 & 0 \\
\pm 1 & 0 & 0 & 0 \\
0 & 0 & 0 & -i \\
0 & 0 & i & 0
\end{array}\right), \Sigma_{ \pm y}=\left(\begin{array}{cccc}
0 & 0 & \pm 1 & 0 \\
0 & 0 & 0 & i \\
\pm 1 & 0 & 0 & 0 \\
0 & -i & 0 & 0
\end{array}\right) \\
\Sigma_{ \pm z}=\left(\begin{array}{cccc}
0 & 0 & 0 & \pm 1 \\
0 & 0 & -i & 0 \\
0 & i & 0 & 0 \\
\pm 1 & 0 & 0 & 0
\end{array}\right)
\end{gathered}
$$

with the commutator $\left[\boldsymbol{\Sigma}_{ \pm}, \boldsymbol{\Sigma}_{\mp}\right]=0$. Also, $\boldsymbol{\Sigma}_{ \pm} \times \boldsymbol{\Sigma}_{ \pm}=2 i \boldsymbol{\Sigma}_{ \pm}$. By complex conjugation of the Sigma matrices in (4), we can see that they obey $\left(\boldsymbol{\Sigma}_{ \pm}\right)^{*}=-\boldsymbol{\Sigma}_{\mp}$. Moreover, the Sigma matrices fulfill, like the Pauli matrices, a metric condition in real space, namely

$$
\Sigma_{ \pm j} \Sigma_{ \pm k}+\Sigma_{ \pm k} \Sigma_{ \pm j}=2 \delta_{j, k} 1_{4}
$$

Thus, the Sigma component matrices squared give unity, and their sum yields $\Sigma_{ \pm}^{2}=31_{4}$. With the help of these matrices, we can reformulate again the four-vector Lorentz transformation and cast it into a form that manifestly shows the $S U(2) \oplus S U$ (2) group structure. When use is made of Eq. (2), and the boost-angle vector $\boldsymbol{\beta}$ and rotation-angle vector $\boldsymbol{\theta}$ are introduced [11], we can write the four-vector Lorentz transformation symmetrically as

$$
\begin{aligned}
\Lambda_{V} & =\exp (i \boldsymbol{\theta} \cdot \mathbf{J}+i \boldsymbol{\beta} \cdot \mathbf{K})=\exp \left(i \boldsymbol{\theta}_{+} \cdot \mathbf{J}_{+}+i \boldsymbol{\theta}_{-} \cdot \mathbf{J}_{-}\right) \\
& =\exp \left(i \boldsymbol{\theta}_{+} \cdot \mathbf{J}_{+}\right) \exp \left(i \boldsymbol{\theta}_{-} \cdot \mathbf{J}_{-}\right),
\end{aligned}
$$

where the definition in Eq. (2) has been inserted, and the new complex angle vectors $\boldsymbol{\theta}_{ \pm}=$ $\boldsymbol{\theta} \mp i \boldsymbol{\beta}=\boldsymbol{\theta}_{\mp}^{*}$ were defined. We also exploited Eq. (3), according to which $\mathbf{J}_{+}$and $\mathbf{J}_{-}$commute, so that their exponential functions can be separated. By help of the Sigma matrices, we can also write $\Lambda_{V}$ as follows

$$
\Lambda_{V}=\exp \left(\frac{i}{2} \boldsymbol{\theta}_{+} \cdot \boldsymbol{\Sigma}_{+}\right) \exp \left(\frac{i}{2} \boldsymbol{\theta}_{-} \cdot \boldsymbol{\Sigma}_{-}\right)=\Lambda_{R} \Lambda_{L} .
$$

The plus sign denotes the right-chiral $(R)$ and the minus sign the left-chiral $(L)$ Lorentz transformation, whereby the affiliation is conventional. When taking the complex conjugate of Eq. (7), we find that $\Lambda_{V}=\Lambda_{V}^{*}$ by means of the properties of the Sigma matrices in Eq. (4). Therefore, the four-vector Lorentz transformation is a real $4 \times 4$ matrix operator, as it should since it operates on a real four-vector $V^{\mu}$ in Minkowski space. In what follows, we will let 
the Lorentz transformation operator act on complex four-vectors that in addition to the spin include the isospin of the fermion.

\section{Dirac equation for an eight-component spinorial wave function}

In this main section, we derive a linear relativistic wave equation in close analogy to the standard Dirac equation, but for a complex eight-component spinor describing a fermion with isospin. We remind the reader that for a free particle of mass $m$ one Casimir operator of the LG [2] is the mass squared, which leads to what is known as the mass-shell condition of the covariant four-momentum $p_{\mu}=(E,-\mathbf{p})$ and reads $p^{\mu} p_{\mu}=m^{2}$. We use standard symbols, notations and conventional units as in the textbooks [9-11] for quantum field theory. Thus, we set Planck's constant to unity and the speed of light $c=1$ and therefore obtain the covariant relativistic quantum-mechanical four-momentum operator as $P_{\mu}=i \partial_{\mu}=$ $\left(i \frac{\partial}{\partial t}, i \frac{\partial}{\partial \mathbf{x}}\right)$. Linearization of the above Casimir operator yields the famous linear wave equation of Dirac that reads

$$
\Gamma^{\mu} i \partial_{\mu} \Phi=m \Phi
$$

The contravariant four-vector Gamma matrix is defined as $\Gamma^{\mu}=\left(\Gamma_{0}, \boldsymbol{\Gamma}\right)$. By squaring the wave Eq. (8), the Gamma matrices are required to obey the Clifford algebra, as needed for Lorentz invariance. So we have subsequently to ensure by appropriate choice that

$$
\Gamma^{\mu} \Gamma^{v}+\Gamma^{v} \Gamma^{\mu}=21_{8} g^{\mu \nu} .
$$

Here, $g^{\mu \nu}$ is the metric tensor in Minkowski space-time in standard notation. When we now square Eq. (8) and use the metric properties of the above Clifford algebra, we retain the Klein-Gordon [15] equation, which is obeyed by each component of the complex spinor function $\Phi$. It has eight components, namely four for the spin doublet and the isospin doublet (corresponding to the real four-vector components of space-time), and two more describing the particle and antiparticle doublet.

We are now making the key step of this section and define the desired Gamma matrices for the extended Dirac equation in terms of the rotation and boost matrix operators $\mathbf{J}$ and $\mathbf{K}$, which we already combined above in the form of the left- and right-chiral spin operator, which was defined in Eq. (2) and written as $\mathbf{J}_{ \pm}=\frac{1}{2} \boldsymbol{\Sigma}_{ \pm}$, with $\mathbf{J}_{ \pm}^{2}=s(s+1) 1_{4}$ and $s=\frac{1}{2}$. Then, it appears most adequate to define the Gamma matrices in the following way

$$
\boldsymbol{\Gamma}=i\left(\begin{array}{cc}
0 & \Delta \boldsymbol{\Sigma}_{-} \\
\Delta \boldsymbol{\Sigma}_{+} & 0
\end{array}\right)
$$

The involved Sigma matrices and their characteristics were already quoted above explicitly. The newly appearing Delta matrix corresponds to the metric in Minkowski space-time and is defined as

$$
\Delta=\left(\begin{array}{cccc}
1 & 0 & 0 & 0 \\
0 & -1 & 0 & 0 \\
0 & 0 & -1 & 0 \\
0 & 0 & 0 & -1
\end{array}\right),
$$

which yields $\Delta^{2}=1_{4}$. Delta has the important property that $\Sigma_{ \pm}=\Delta \Sigma_{\mp} \Delta$, which guarantees that $\Gamma_{j}=-1_{8}$, and thus $\Gamma^{2}=-31_{8}$, and consequently that the component matrices $\Gamma_{j}$ anticommute and therefore obey the Clifford algebra.

In order to obtain the other Gamma matrices in the Weyl basis, we are free to first define the so-called matrix $\Gamma_{5}=i \Gamma_{0} \Gamma_{x} \Gamma_{y} \Gamma_{z}$, from which then by solving for $\Gamma_{0}$ we obtain that latter 
matrix itself by exploiting the above-defined vector Gamma matrix $\boldsymbol{\Gamma}$. In the conventional Weyl basis [9-11], the matrix $\Gamma_{5}$ is given by

$$
\Gamma_{5}=\left(\begin{array}{cc}
-1_{4} & 0 \\
0 & 1_{4}
\end{array}\right) \text {. }
$$

After some straightforward but tedious algebra, we obtain

$$
\Gamma_{0}=\left(\begin{array}{cc}
0 & i \Delta \\
-i \Delta & 0
\end{array}\right),
$$

which obeys $\left(\Gamma_{0}\right)^{2}=1_{8}$ and mutually anticommutes with the four other Gamma matrices by its definition. These derivations complete the definitions of the relevant Gamma matrices, which are the key new ingredients of the linear wave Eq. (8). By definition, we have $\Gamma^{\mu} \Gamma_{\mu}=$ $\left(\Gamma_{0}\right)^{2}-\Gamma^{2}=41_{8}$.

We may in the end discuss the important chiral projection operator $P_{ \pm}=\frac{1}{2}\left(1 \pm \Gamma_{5}\right)$, which is idempotent and has the effect that $P_{ \pm} \Gamma^{\mu}=\Gamma^{\mu} P_{\mp}$, i.e., its sign switches by commutation with the Gamma matrices. Using the Weyl basis has the big advantage that the wave equation in the limit of a vanishing fermion mass decouples into the left- and right-chiral components, a convenient property well known from the standard Dirac equation in that basis.

The spinorial generators of the Lorentz group [9-11] can generally be written as

$$
M^{\mu \nu}=\frac{i}{4}\left[\Gamma^{\mu}, \Gamma^{\nu}\right]=\left(\begin{array}{cccc}
0 & R_{x} & R_{y} & R_{z} \\
-R_{x} & 0 & S_{z} & -S_{y} \\
-R_{y} & -S_{z} & 0 & S_{x} \\
-R_{z} & S_{y} & -S_{x} & 0
\end{array}\right) .
$$

Here, the rapidity (boost) operator is defined as

$$
\mathbf{R}=\frac{i}{2} \Gamma_{0} \boldsymbol{\Gamma}=\frac{1}{2}\left(\begin{array}{cc}
0 & -\Delta \\
\Delta & 0
\end{array}\right) \boldsymbol{\Gamma},
$$

and similarly the spin (rotation) operator as

$$
S_{x}=\frac{i}{2} \Gamma_{y} \Gamma_{z}=\frac{-i}{2}\left(\begin{array}{cc}
\Sigma_{+y} \Sigma_{+z} & 0 \\
0 & \Sigma_{-y} \Sigma_{-z}
\end{array}\right), \quad \text { (cyclic). }
$$

By insertion of the expressions for the Gamma matrices into the defining Eqs. (15) and (16), one obtains

$$
\begin{gathered}
\mathbf{S}=\left(\begin{array}{cc}
\mathbf{S}_{R} & 0 \\
0 & \mathbf{S}_{L}
\end{array}\right)=\frac{1}{2}\left(\begin{array}{cc}
\Sigma_{+} & 0 \\
0 & \Sigma_{-}
\end{array}\right) . \\
\mathbf{R}=\left(\begin{array}{cc}
\mathbf{R}_{R} & 0 \\
0 & \mathbf{R}_{L}
\end{array}\right)=\left(\begin{array}{cc}
-i \mathbf{S}_{R} & 0 \\
0 & i \mathbf{S}_{L}
\end{array}\right) .
\end{gathered}
$$

So the rapidity operator is fully determined by the spin operator, which consists of the rightchiral spin $\mathbf{S}_{R}$ and left-chiral spin $\mathbf{S}_{L}$, which yields $\mathbf{R}_{R, L}=\mp i \mathbf{S}_{R, L}$. Consequently, both pairs of matrix vectors obey the Lorentz algebra like the boost and rotation vectors.

Applying the rules of anticommutation of the Gamma matrices according to the Clifford algebra given by Eq. (9) and the definitions in Eqs. (15) and (16), we find after considerable algebra that $\mathbf{S}$ and $\mathbf{R}$ also obey the Lorentz algebra, i.e., we have $\mathbf{S} \times \mathbf{S}=i \mathbf{S}, \mathbf{R} \times \mathbf{R}=-i \mathbf{S}$, and $\mathbf{S} \times \mathbf{R}=i \mathbf{R}$. We can see that $\mathbf{S}$ is Hermitian and corresponds to the rotation operator $\mathbf{J}$, and $\mathbf{R}$ is anti-Hermitian and corresponds to the boost operator $\mathbf{K}$ of the four-vector Lorentz 
group generators. Consequently, we can define the right- and left-chiral spinorial rotation operator as

$$
\mathbf{S}_{ \pm}=\frac{1}{2}(\mathbf{S} \pm i \mathbf{R})
$$

By exploiting the definitions in Eqs. (15) and (16) or using the results in Eqs. (17) and (18), we find that

$$
\mathbf{S}_{ \pm}=\mathbf{S} P_{ \pm}
$$

involving the projector operator. In analogy to Eq. (3) they obey the Lorentz algebra

$$
\mathbf{S}_{ \pm} \times \mathbf{S}_{ \pm}=i \mathbf{S}_{ \pm}, \mathbf{S}_{ \pm} \times \mathbf{S}_{\mp}=0 .
$$

When making use of the boost angle vector $\boldsymbol{\beta}$ and rotation angle vector $\boldsymbol{\theta}[9,11]$, we can generally write the spinorial Lorentz transformation as

$$
\Lambda_{S}=\exp (i \boldsymbol{\theta} \cdot \mathbf{S}+i \boldsymbol{\beta} \cdot \mathbf{R}) \text {. }
$$

Note that $\left[\Gamma_{0}, \mathbf{S}\right]=0,\left\{\Gamma_{0}, \mathbf{R}\right\}=0, \mathbf{S}^{\dagger}=\mathbf{S}$, and $\mathbf{R}^{\dagger}=-\mathbf{R}$. As a result, the inverse Lorentz transformation reads $\Lambda_{S}^{-1}=\Gamma_{0} \Lambda_{S}^{\dagger} \Gamma_{0}$, which just gives a minus sign in front of the exponents in Eq. (22). When use is made of the right- and left-chiral rotation operators $\mathbf{S}_{ \pm}$, we can also write the spinorial Lorentz transformation as

$$
\Lambda_{S}=\exp \left(i \boldsymbol{\theta}_{+} \cdot \mathbf{S}_{+}+i \boldsymbol{\theta}_{-} \cdot \mathbf{S}_{-}\right)=\exp \left(i \boldsymbol{\theta}_{+} \cdot \mathbf{S}_{+}\right) \exp \left(i \boldsymbol{\theta}_{-} \cdot \mathbf{S}_{-}\right)
$$

involving the complex angle vectors $\boldsymbol{\theta}_{ \pm}=\boldsymbol{\theta}_{\mp}^{*}$. We thereby exploited that $\mathbf{S}_{+}$and $\mathbf{S}_{-}$commute, so that the exponential functions can be separated. By use of relation (19), and using that $P_{+}+P_{-}=1_{8}$, and the idempotence of the projectors, we can finally write

$$
\Lambda_{S}=\exp \left(i \boldsymbol{\theta}_{+} \cdot \mathbf{S}\right) P_{+}+\exp \left(i \boldsymbol{\theta}_{-} \cdot \mathbf{S}\right) P_{-} .
$$

For zero boost angle $\boldsymbol{\beta}=\mathbf{0}$, we simply get for $\Lambda$ the spinorial rotation as determined by the operator $\exp (i \boldsymbol{\theta} \cdot \mathbf{S})$, corresponding to Wigner's little group [2,3]. Although $\mathbf{S}$ is Hermitian, the Lorentz transformation is not, simply because of the complex angle vectors $\boldsymbol{\theta}_{ \pm}$. These considerations are all rather general. Upon insertion of the specific results from Eqs. (17) and (18), we obtain the block-diagonal Lorentz transformation in the form

$$
\Lambda_{S}=\left(\begin{array}{cc}
\Lambda_{R} & 0 \\
0 & \Lambda_{L}
\end{array}\right)=\left(\begin{array}{cc}
\exp \left(i \boldsymbol{\theta}_{+} \cdot \mathbf{S}_{R}\right) & 0 \\
0 & \exp \left(i \boldsymbol{\theta}_{-} \cdot \mathbf{S}_{L}\right)
\end{array}\right)
$$

This acts on the bi-spinor $\Phi^{\dagger}=\left(\phi_{R}^{\dagger}, \phi_{L}^{\dagger}\right)$. The two elements of the $2 \times 2$ matrix $\Lambda_{S}$ are identical with the two factors appearing in the vectorial Lorentz transformation $\Lambda_{V}$ in (7).

\section{The Lagrangian density in the Weyl basis}

The Lorentz-transformed spinor wave function is named as $\Phi_{\Lambda}=\Lambda \Phi$. We define as usual the conjugate spinor as $\bar{\Phi}=\left(\Gamma_{0} \Phi\right)^{\dagger}=\Phi^{\dagger} \Gamma_{0}$. Then, it is easy, by following the comments made after Eq. (22), to show the Lorentz invariance of the mass term, i.e., that $\bar{\Phi}_{\Lambda} \Phi_{\Lambda}=\bar{\Phi} \Phi$. Similarly, the kinetic term can be shown to covariant. The Lagrangian density therefore reads

$$
\mathcal{L}_{k}=\bar{\Phi}\left(\Gamma^{\mu} i \partial_{\mu} \Phi-m \Phi\right) .
$$

This is formally identical with that of the standard Dirac equation, yet here it refers to a fermion field $\Phi$ that includes isospin at the outset and of course has the particle and antiparticle 
doublet and, respectively, two spin components. As usually, the variation in $\mathcal{L}$ with respect to the adjoint spinor $\bar{\Phi}$ yields the linear wave Eq. (8). The Gamma matrices were already given in Eqs. (10), (12) and (13). Note that according to its above definition the adjoint field reads

$$
\bar{\Phi}=\Phi^{\dagger} \Gamma_{0}=\left(-\phi_{L}^{\dagger} i \Delta, \phi_{R}^{\dagger} i \Delta\right) \text {. }
$$

As we are working in the Weyl basis, the above Lagrangian can also be written more explicitly in terms of the right- and left-chiral fields as

$$
\begin{aligned}
\mathcal{L} & =\mathcal{L}_{k}+\mathcal{L}_{m} \\
& =-\phi_{R}^{\dagger} \Sigma_{R}^{\mu} i \partial_{\mu} \phi_{R}+\phi_{L}^{\dagger} \Sigma_{L}^{\mu} i \partial_{\mu} \phi_{L}-m\left(\phi_{R}^{\dagger} i \Delta \phi_{L}-\phi_{L}^{\dagger} i \Delta \phi_{R}\right)
\end{aligned} .
$$

Here, we renamed the spin matrices as follows $\Sigma_{R, L}=\Sigma_{ \pm}$and introduced the contravariant four-vector matrices

$$
\Sigma_{R, L}^{\mu}=\left(\mp 1_{4}, \Sigma_{ \pm}\right)
$$

Correspondingly, we obtain the Gamma matrices in the concise matrix form

$$
\Gamma^{\mu}=\left(\begin{array}{cc}
0 & \Gamma_{L}^{\mu} \\
\Gamma_{R}^{\mu} & 0
\end{array}\right)=\left(\begin{array}{cc}
0 & i \Delta \Sigma_{L}^{\mu} \\
i \Delta \Sigma_{R}^{\mu} & 0
\end{array}\right) .
$$

Then, the two coupled Weyl equations [16] for the fermion with isospin read

$$
\begin{aligned}
& \Gamma_{R}^{\mu} i \partial_{\mu} \phi_{R}=m \phi_{L}, \\
& \Gamma_{L}^{\mu} i \partial_{\mu} \phi_{L}=m \phi_{R} .
\end{aligned}
$$

Written out more explicitly, we obtain

$$
\begin{aligned}
& \left(+1_{4} \frac{\partial}{\partial t}-\Sigma_{R} \cdot \frac{\partial}{\partial \mathbf{x}}\right) \phi_{R}=m \Delta \phi_{L}, \\
& \left(-1_{4} \frac{\partial}{\partial t}-\boldsymbol{\Sigma}_{L} \cdot \frac{\partial}{\partial \mathbf{x}}\right) \phi_{L}=m \Delta \phi_{R} .
\end{aligned}
$$

The advantage of the Weyl basis becomes obvious here, since for $m=0$ the equations become decoupled. By help of relation $\Delta \boldsymbol{\Sigma}_{R, L}=\Sigma_{L, R} \Delta$, we retain after operation of the corresponding differential operator (with the opposite sign in front of the time derivate) on each of the above equations the Klein-Gordon $[15,17]$ equation

$$
\left(\frac{\partial^{2}}{\partial t^{2}}-\frac{\partial^{2}}{\partial \mathbf{x}^{2}}+m^{2}\right) \phi_{R, L}=0 .
$$

Hereby, we used the property of the Sigma matrix that for any three-vector $\mathbf{A}$ we have $\left(\boldsymbol{\Sigma}_{R, L} \cdot \mathbf{A}\right)^{2}=\mathbf{A}^{2} 1_{4}$. In Eqs. (31) and (32), the left- and right-chiral fields appear in a rather equivalent form with their respective Gamma and Sigma matrices, which both refer to the same basis in the complex four-vector space.

Again, by means of the relation $\Delta \Sigma_{R, L}=\Sigma_{L, R} \Delta$ one can without loss of generality decide to use either one of them, e.g., we just take $\Sigma_{R}$. When renaming the fields as follows $\phi_{R}=\tilde{\phi}_{R}$ and $i \Delta \phi_{L}=\tilde{\phi}_{L}$, which corresponds to the contravariant version of the field in the language of tensors in Minkowski space given the metric nature of the $\Delta$ matrix in Eq. (13), we obtain the Weyl equations in a form more familiar from the standard Dirac equation as follows

$$
\begin{aligned}
& i\left(\frac{\partial}{\partial t}-\boldsymbol{\Sigma}_{R} \cdot \frac{\partial}{\partial \mathbf{x}}\right) \tilde{\phi}_{R}=m \tilde{\phi}_{L}, \\
& i\left(\frac{\partial}{\partial t}+\boldsymbol{\Sigma}_{R} \cdot \frac{\partial}{\partial \mathbf{x}}\right) \tilde{\phi}_{L}=m \tilde{\phi}_{R} .
\end{aligned}
$$

Here, we omitted the unit matrix $1_{4}$ to ease the notation. Squaring those equations of course reproduces the above Klein-Gordon equation for each of the eight spinor components. 
By changing the vector basis, the $\Sigma_{R}$ matrix can be written in a form in which the $z$ component becomes diagonal. The result is given in "Appendix." We call this matrix after the unitary transformation $\tilde{\Sigma}_{R}$. It can then be written concisely in terms of the three wellknown Pauli matrices in block-diagonal tensor-product form as

$$
\tilde{\boldsymbol{\Sigma}}_{R}=\left(\left(\begin{array}{cc}
\sigma_{x} & 0 \\
0 & \sigma_{x}
\end{array}\right),\left(\begin{array}{cc}
\sigma_{y} & 0 \\
0 & \sigma_{y}
\end{array}\right),\left(\begin{array}{cc}
\sigma_{z} & 0 \\
0 & \sigma_{z}
\end{array}\right)\right)=1_{2} \otimes \sigma .
$$

Apparently, the above Weyl equations can then be further decomposed in the following way. We denote the spinor in the new basis again as $\Phi$ and can reassemble the spinor components of $\Phi^{\dagger}=\left(\phi_{R}^{\dagger}, \phi_{L}^{\dagger}\right)=\left(\phi_{1}, \phi_{2}, \phi_{3}, \phi_{4}, \phi_{5}, \phi_{6}, \phi_{7}, \phi_{8}\right)^{\dagger}$ in such a way that the subsequent four pairs can be constructed:

$$
\psi_{R}=\left(\begin{array}{c}
\phi_{1} \\
\phi_{2}
\end{array}\right), \quad \psi_{L}=\left(\begin{array}{c}
\phi_{5} \\
\phi_{6}
\end{array}\right), \quad \chi_{R}=\left(\begin{array}{c}
\phi_{3} \\
\phi_{4}
\end{array}\right), \quad \chi_{L}=\left(\begin{array}{c}
\phi_{7} \\
\phi_{8}
\end{array}\right) .
$$

They obey two independent sets of standard Weyl equations. The first is obtained as

$$
\begin{aligned}
& i\left(\frac{\partial}{\partial t}-\sigma \cdot \frac{\partial}{\partial \mathbf{x}}\right) \psi_{R}=m \psi_{L}, \\
& i\left(\frac{\partial}{\partial t}+\sigma \cdot \frac{\partial}{\partial \mathbf{x}}\right) \psi_{L}=m \psi_{R} .
\end{aligned}
$$

Similarly, one obtains for the second

$$
\begin{aligned}
& i\left(\frac{\partial}{\partial t}-\sigma \cdot \frac{\partial}{\partial \mathbf{x}}\right) \chi_{R}=m \chi_{L}, \\
& i\left(\frac{\partial}{\partial t}+\sigma \cdot \frac{\partial}{\partial \mathbf{x}}\right) \chi_{L}=m \chi_{R} .
\end{aligned}
$$

We again omitted here the unit matrix $1_{2}$ to ease the notation.

\section{Chiral symmetry and $S U(2)$ gauge theory}

We continue the general discussion of the extended Dirac equation and look into the property of chirality in more detail. We return to the extended Dirac Eq. (8), but can write it after unitary transformation in the Weyl basis in a new form with the contravariant Gamma matrix derived from Eq. (34), but now with the Sigma matrix of Eq. (35).

$$
\tilde{\Gamma}^{\mu}=\left(\tilde{\Gamma}_{0}, \tilde{\boldsymbol{\Gamma}}_{R}\right)=\left(\left(\begin{array}{cc}
0 & 1_{4} \\
1_{4} & 0
\end{array}\right),\left(\begin{array}{cc}
0 & \tilde{\boldsymbol{\Sigma}}_{R} \\
-\tilde{\boldsymbol{\Sigma}}_{R} & 0
\end{array}\right)\right) .
$$

Then, matrix $\tilde{\Gamma}_{5}$ is equal to minus $\Gamma_{5}$, which is given in Eq. (12). It is well known that chiral symmetry is violated by the mass term, since $\tilde{\Gamma}_{5}$ anticommutes with $\tilde{\Gamma}^{\mu}$, but is remains valid for $m=0$. In that case, the spinor $\Phi$ may have a general phase which leaves the massless Lagrangian invariant. The related phase operator may be written

$$
P(\alpha, \lambda)=\exp i\left(g^{\prime} \alpha 1_{8}+g \lambda \tilde{\Gamma}_{5}\right) .
$$

The coupling constants are $g^{\prime}$ for electromagnetism and $g$ for chirality, and the corresponding phase angles are $\alpha$ and $\lambda$. The massless kinetic Lagrangian after Eq. (26) then reads

$$
\mathcal{L}=\Phi^{\dagger} \tilde{\Gamma}_{0} \tilde{\Gamma}^{\mu} i \partial_{\mu} \Phi
$$

As $\tilde{\Gamma}_{5}$ is Hermitian, the phase operator is unitary and obeys $P^{\dagger} P=1$. Moreover, $P$ commutes with the kinetic term that involves the Gammas only quadratically, with which $\tilde{\Gamma}_{5}$ of course commutes. So the phase factor in Eq. (40) is compatible with chiral symmetry and does not break it. 
In addition to chirality, we find as a new and important feature that there is full $S U$ (2) symmetry as an internal symmetry related to the field equation of the fermion. To discuss this property more generally, we go back to Eq. (34). In addition to the spin associated with the matrix vector $\tilde{\boldsymbol{\Sigma}}_{R}$ we still have the spin associated with the matrix vector $\tilde{\boldsymbol{\Sigma}}_{L}$, which both are given explicitly in "Appendix." Accordingly, we can write the latter concisely as the following tensor product

$$
\tilde{\Sigma}_{L}=\sigma \otimes 1_{2}
$$

The subsequent $8 \times 8$ matrix can thus be defined

$$
\boldsymbol{\Sigma}=\left(\begin{array}{cc}
\tilde{\boldsymbol{\Sigma}}_{L} & 0 \\
0 & \tilde{\boldsymbol{\Sigma}}_{L}
\end{array}\right)=1_{2} \otimes \tilde{\boldsymbol{\Sigma}}_{L}
$$

It obeys $\Sigma^{2}=31_{8}$ and has the same algebraic properties as the Pauli matrices. It is important to keep in mind that componentwise the following commutators hold

$$
[\Sigma, \tilde{\boldsymbol{\Gamma}}]=0, \quad\left[\Sigma, \tilde{\Gamma}_{0}\right]=0 .
$$

Consequently, we can construct the adequate phase operator reflecting the combined $U(1)$ and $S U(2)$ symmetries as follows

$$
P(\alpha, \lambda)=\exp \frac{i}{2}\left(g^{\prime} \alpha(x) 1_{8}+g \lambda(x) \cdot \Sigma\right) .
$$

It operates on the chiral doublet $\Phi^{\dagger}=\left(\phi_{R}^{\dagger}, \phi_{L}^{\dagger}\right)$. Here, the Sigma matrices appear as a nonfundamental representation of $S U(2)$, but they make essential use of the Pauli matrices. Lambda now is a three-vector of complex numbers. The coupling constants are $g^{\prime}$, again related to electromagnetism, and $g$, related to what looks like the weak interactions, if we transit to gauge theory. That means we let $\alpha(x)$ and $\lambda(x)$ be functions of the space-time coordinate $x$ as an abbreviation for $x^{\mu}=(t, \mathbf{x})$. Let us continue by discussing the case of a massive fermion doublet. We consider the extended Dirac Eq. (8) again with the Gamma matrices as given in Eq. (39).

Now the coupling to gauge fields in the Dirac equation conventionally is achieved by minimal substitution via the covariant derivate $D^{\mu}=\partial^{\mu}-V^{\mu}$, with the interaction gauge fields being $B^{\mu}$ for the $U(1)$ hypercharge gauge group and three-vector $\mathbf{W}^{\mu}$ for the $S U(2)$ gauge group. All this is adding up to an expression for the coupling term which can be written as a matrix tensor product $V^{\mu}=1_{2} \otimes U^{\mu} \otimes 1_{2}$ with

$$
U^{\mu}(x)=\frac{i}{2}\left(g^{\prime} B^{\mu}(x) 1_{2}+g \mathbf{W}^{\mu}(x) \cdot \sigma\right) .
$$

We do not present further any detailed derivations, since the subsequent procedure is well known from the Standard Model (SM) of elementary particle physics [9-11]. After rotation of the gauge fields and by fixing the Weinberg angle, we obtain the electromagnetic field $A^{\mu}$ and weak boson field $Z^{\mu}$, together with which the total gauge coupling field reads

$$
\begin{aligned}
U^{\mu}(x)= & i e\left(\begin{array}{ll}
1 & 0 \\
0 & 0
\end{array}\right) A^{\mu}+\frac{i}{2} \sqrt{g^{\prime 2}+g^{2}}\left(\begin{array}{cc}
1 & 0 \\
0 & -1
\end{array}\right) Z^{\mu}(x) \\
& +\frac{i}{2} g\left(\begin{array}{ll}
0 & 1 \\
0 & 0
\end{array}\right) W_{-}^{\mu}(x)+\frac{i}{2} g\left(\begin{array}{ll}
0 & 0 \\
1 & 0
\end{array}\right) W_{+}^{\mu}(x) .
\end{aligned}
$$

Here, $e=g g^{\prime} / \sqrt{g^{\prime 2}+g^{2}}$ is the electric charge unit ( $-e$ for the electron charge), in terms of the coupling constants, and the complex gauge fields are $W_{ \pm}^{\mu}=W_{x}^{\mu} \pm i W_{y}^{\mu}$. After this known 
procedure of $S U$ (2) symmetry breaking, the charged electron and the uncharged neutrino emerge naturally. The fields $W_{ \pm}^{\mu}$ describe charge exchange and transmute the electron into a neutrino and vice versa the neutrino into an electron. As a result, the flavor doublet electron and neutrino originate via breaking of the internal $S U$ (2) from the original fermion doublet based on the vector representation of the Lorentz group.

\section{Discussion and conclusion}

In conclusion, Eq. (34) based on the vector representation of the Lorentz group is after an adequate unitary transformation equivalent to the two standard Dirac equations, namely Eqs. (37) and (38), with the same mass. Both sets can equally describe a fermion doublet and thus seem to be appropriate for representing the empirically suggested and experimentally corroborated assemblage of fermions as lepton and quark doublets. This applies to all three families occurring in the SM, in which each fermion is described by the standard Dirac equation.

For zero mass, the eight-component spinor field $\Phi$ can be broken down into the four independent elementary Weyl fields $\psi_{R, L}$ and $\chi_{R, L}$. However, as the two fermions in the doublet are assumed here to have the same mass, the problem of the large measured mass difference, e.g., between the electrons and neutrinos, cannot be addressed. Admittedly, the difficult subject of the origin of mass (or of the striking differences in the real fermion masses) is way beyond the scope of this paper.

It appears from the present analysis, doubling the standard Dirac equation from four-spinor to eight-spinor form by help of the vector representation of the Lorentz group, that one obtains an isospin-type doublet naturally (for a given generation), and that by mixing via the intrinsic $S U$ (2) symmetry both fermions in the doublet are at the outset unitarily equivalent. Also chiral symmetry is valid in the massless case. After $S U(2)$ symmetry breaking, as obtained by following the construction of the Glashow-Weinberg-Salam model, the electric charges and electromagnetic interaction field $A^{\mu}$, as well as the weak interactions mediated by the $Z^{\mu}$ and $W_{ \pm}^{\mu}$ boson fields emerge naturally. In this way, one obtains the electroweak properties of the lepton doublets, like that of the electron and neutrino, or of the quark doublets like that of the up- and down-quark.

Funding Open Access funding enabled and organized by Projekt DEAL.

Open Access This article is licensed under a Creative Commons Attribution 4.0 International License, which permits use, sharing, adaptation, distribution and reproduction in any medium or format, as long as you give appropriate credit to the original author(s) and the source, provide a link to the Creative Commons licence, and indicate if changes were made. The images or other third party material in this article are included in the article's Creative Commons licence, unless indicated otherwise in a credit line to the material. If material is not included in the article's Creative Commons licence and your intended use is not permitted by statutory regulation or exceeds the permitted use, you will need to obtain permission directly from the copyright holder. To view a copy of this licence, visit http://creativecommons.org/licenses/by/4.0/.

\section{Appendix A: various relevant matrices}

For the sake of completeness, we firstly quote the component matrices of the Hermitian rotation three-vector $\mathbf{J}$, which is the generator of the $S O$ (3) rotation subgroup of the Lorentz 
group. We obtain the component matrices as

$$
J_{x}=\left(\begin{array}{cccc}
0 & 0 & 0 & 0 \\
0 & 0 & 0 & 0 \\
0 & 0 & 0 & -i \\
0 & 0 & i & 0
\end{array}\right), J_{y}=\left(\begin{array}{cccc}
0 & 0 & 0 & 0 \\
0 & 0 & 0 & i \\
0 & 0 & 0 & 0 \\
0 & -i & 0 & 0
\end{array}\right), J_{z}=\left(\begin{array}{cccc}
0 & 0 & 0 & 0 \\
0 & 0 & -i & 0 \\
0 & i & 0 & 0 \\
0 & 0 & 0 & 0
\end{array}\right) .
$$

For the absolute value of the rotation operator, we have the matrix

$$
J_{x}^{2}+J_{y}^{2}+J_{z}^{2}=\left(\begin{array}{llll}
0 & 0 & 0 & 0 \\
0 & 2 & 0 & 0 \\
0 & 0 & 2 & 0 \\
0 & 0 & 0 & 2
\end{array}\right)
$$

The component matrices of $\mathbf{K}$ are also quoted here for completeness

$$
K_{x}=\left(\begin{array}{cccc}
0 & -i & 0 & 0 \\
-i & 0 & 0 & 0 \\
0 & 0 & 0 & 0 \\
0 & 0 & 0 & 0
\end{array}\right), K_{y}=\left(\begin{array}{cccc}
0 & 0 & -i & 0 \\
0 & 0 & 0 & 0 \\
-i & 0 & 0 & 0 \\
0 & 0 & 0 & 0
\end{array}\right), K_{z}=\left(\begin{array}{cccc}
0 & 0 & 0 & -i \\
0 & 0 & 0 & 0 \\
0 & 0 & 0 & 0 \\
-i & 0 & 0 & 0
\end{array}\right)
$$

It is then straightforward to obtain the matrix

$$
K_{x}^{2}+K_{y}^{2}+K_{z}^{2}=\left(\begin{array}{cccc}
-3 & 0 & 0 & 0 \\
0 & -1 & 0 & 0 \\
0 & 0 & -1 & 0 \\
0 & 0 & 0 & -1
\end{array}\right)
$$

Consequently, we find $\mathbf{J}^{2}-\mathbf{K}^{2}=31_{4}$.

The matrices we considered above and in the main text of the paper were based on the standard vector basis in four-dimensional space. The basis vectors are

$$
\varphi_{1}=\left(\begin{array}{l}
1 \\
0 \\
0 \\
0
\end{array}\right), \varphi_{2}=\left(\begin{array}{l}
0 \\
1 \\
0 \\
0
\end{array}\right) \varphi_{3}=\left(\begin{array}{l}
0 \\
0 \\
1 \\
0
\end{array}\right), \varphi_{4}=\left(\begin{array}{l}
0 \\
0 \\
0 \\
1
\end{array}\right) .
$$

The matrices $\Sigma_{R z}$ and $\Sigma_{L z}$ become diagonal in the new basis, which can be made orthonormal by multiplication with the factor $\frac{1}{\sqrt{2}}$. The basis vectors read

$$
\chi_{1}=\left(\begin{array}{l}
1 \\
0 \\
0 \\
1
\end{array}\right), \chi_{2}=\left(\begin{array}{c}
0 \\
1 \\
-i \\
0
\end{array}\right) \quad \chi_{3}=\left(\begin{array}{l}
0 \\
1 \\
i \\
0
\end{array}\right), \chi_{4}=\left(\begin{array}{c}
1 \\
0 \\
0 \\
-1
\end{array}\right) \text {. }
$$

The Sigma matrix (corresponding to $\boldsymbol{\Sigma}_{+}$introduced in the main text) can, in this more appropriate basis, be written as

$$
\tilde{\boldsymbol{\Sigma}}_{R}=\left(\left(\begin{array}{llll}
0 & 1 & 0 & 0 \\
1 & 0 & 0 & 0 \\
0 & 0 & 0 & 1 \\
0 & 0 & 1 & 0
\end{array}\right),\left(\begin{array}{cccc}
0 & -i & 0 & 0 \\
i & 0 & 0 & 0 \\
0 & 0 & 0 & -i \\
0 & 0 & i & 0
\end{array}\right),\left(\begin{array}{cccc}
1 & 0 & 0 & 0 \\
0 & -1 & 0 & 0 \\
0 & 0 & 1 & 0 \\
0 & 0 & 0 & -1
\end{array}\right)\right) .
$$


It obeys the angular momentum algebra and the metric condition like in Eq. (5). The Sigma matrix (corresponding to $\Sigma_{-}$introduced in the main text) can, in the new basis, be written as

$$
\tilde{\boldsymbol{\Sigma}}_{L}=\left(\left(\begin{array}{cccc}
0 & 0 & 0 & -1 \\
0 & 0 & -1 & 0 \\
0 & -1 & 0 & 0 \\
-1 & 0 & 0 & 0
\end{array}\right),\left(\begin{array}{cccc}
0 & 0 & 0 & -i \\
0 & 0 & i & 0 \\
0 & -i & 0 & 0 \\
i & 0 & 0 & 0
\end{array}\right),\left(\begin{array}{cccc}
-1 & 0 & 0 & 0 \\
0 & -1 & 0 & 0 \\
0 & 0 & 1 & 0 \\
0 & 0 & 0 & 1
\end{array}\right)\right) \text {. }
$$

These two Sigma matrix vectors in the new basis do of course also commute componentwise. They can be concisely written as $\tilde{\Sigma}_{R}=1_{2} \otimes \sigma$ and similarly $\tilde{\Sigma}_{L}=\sigma \otimes 1_{2}$. The original expressions for the $x$ - and $z$-components in Eq. (A.8) can be replaced by their negative values, yielding the above tensor-product form, which by the way is consistent with the tensor product of the $S U(2) \times S U(2)$ representation of the Lorentz group.

\section{References}

1. P.A.M. Dirac, Relativistic wave equations. Proc. R. Soc. Lond. Ser. A Math. Phys. Sci. 155, 886 (1936)

2. E. Wigner, On unitary representations of the inhomogeneous lorentz group. Ann. Math. Second Ser. 40(1), 149 (1939). https://doi.org/10.2307/1968551

3. V. Bargman, E. Wigner, Group theoretical discussion of relativistic wave equations. Proc. N.A.S. 34, 211 (1948)

4. H. Joos, Zur Darstellungstheorie der inhomogenen Lorentzgruppe als Grundlage quantenmechanischer Kinematik. Fortschritte der Physik 10, 65 (1962)

5. P.A.M. Dirac, The quantum theory of the electron. Proc. R. Soc. Lond. Ser. A Math. Phys. Sci. 117, 610 (1928). https://doi.org/10.1098/rspa.1928.0023

6. S. Esposito, Searching for an equation: Dirac, Majorana and the others. Ann. Phys. 327, 1617 (2012)

7. I.M. Benn, R.W. Tucker, Fermions without spinors. Commun. Math. Phys. 89, 341 (1983)

8. E. Kähler, Der innere Differentialkalkül. Rend. Mat. 21(3-4), 425 (1962)

9. M. Kaku, Quantum Field Theory, A Modern Introduction (Oxford University Press, New York, 1993)

10. M.E. Peskin, D.V. Schroeder, An Introduction to Quantum Field Theory (Addison-Wesley Publishing Company, Reading, 1995)

11. M.D. Schwartz, Quantum Field Theory and the Standard Model (Cambridge University Press, Cambridge, 2014)

12. R. Kerner, J. Lukierski, $Z_{3}$-graded colour Dirac equations for quarks, confinement and generalized Lorentz symmetries. Phys. Lett. B792, 233 (2019)

13. R. Kerner, The quantum nature of Lorentz invariance. Universe 5(1), 1 (2019)

14. W. Pauli, Zur Quantenmechanik des magnetischen Elektrons. Z. Physik 43, 601 (1927)

15. O. Klein, Elektrodynamik und Wellenmechanik vom Standpunkt des Korrespondenzprinzips. Z. Physik 42, 407 (1926)

16. H. Weyl, Elektron und Gravitation. Z. Physik 56, 330 (1929)

17. W. Pauli, V. Weisskopf, Über die Quantisierung der skalaren relativistischen Wellengleichung. Helv. Phys. Acta 7, 709 (1934) 